\title{
EEG- and EOG-based Asynchronous Hybrid BCI: A System Integrating a Speller, a Web Browser, an E-mail Client, and a File Explorer
}

\author{
Shenghong $\mathrm{He}^{1}$, Yajun Zhou ${ }^{1}$, Tianyou Yu${ }^{1}$, Rui Zhang ${ }^{2}$, Qiyun Huang ${ }^{1}$, Lin Chuai ${ }^{3}$, Madah-Ul-Mustafa ${ }^{1}$, \\ Zhenghui $\mathrm{Gu}^{1}$, Zhu Liang $\mathrm{Yu}^{1}$, Huiling $\mathrm{Tan}^{4,5}$ and Yuanqing $\mathrm{Li}^{1, *}$, Fellow, IEEE
}

\begin{abstract}
This paper presents a new asynchronous hybrid brain-computer interface (BCI) system that integrates a speller, a web browser, an e-mail client, and a file explorer using electroencephalographic (EEG) and electrooculography (EOG) signals. More specifically, an EOG-based button selection method, which requires the user to blink his/her eyes synchronously with the target button's flashes during button selection, is first presented. Next, we propose a mouse control method by combining EEG and EOG signals, in which the left-/right-hand motor imagery (MI)related EEG is used to control the horizontal movement of the mouse and the blink-related EOG is used to control the vertical movement of the mouse and to select/reject a target. These two methods are further combined to develop the integrated hybrid BCI system. With the hybrid BCI, users can input text, access the internet, communicate with others via e-mail, and manage files in their computer using only EEG and EOG without any body movements. Ten healthy subjects participated in a comprehensive online experiment, and superior performance was achieved compared with our previously developed P300- and MI-based BCI and some other asynchronous BCIs, therefore demonstrating the system's effectiveness.
\end{abstract}

Index Terms-Electroencephalography (EEG), electrooculography (EOG), speller, web browser, e-mail client, file explorer.

\section{INTRODUCTION}

A human-machine interface (HMI) system is a communication system that allows users to interact with external devices [1]. In recent years, biological signals, such as electroencephalography (EEG) and electrooculography (EOG), have been widely used to augment or as alternatives to traditional HMIs (such as

This work was supported by The National Key Research and Development Program of China under Grant 2017YFB1002505, the National Natural Science Foundation of China under Grant 61633010 and 61703101, the Guangdong Natural Science Foundation under Grant 2014A030312005, and the Key R\&D Program of Guangdong Province, China under grant 2018B030339001.

${ }^{1}$ School of Automation Science and Engineering, South China University of Technology, and also with the Guangzhou Key Laboratory of Brain Computer Interface and Applications (no. 15180006), Guangzhou 510640, China.

${ }^{2}$ School of Electrical Engineering \& Intelligentization, Dongguan University of Technology, Dongguan 523808, China.

${ }^{3}$ Faculty of Brain Sciences, University College London, London WC1E 6BT, UK.

${ }^{4}$ Medical Research Council Brain Network Dynamics Unit at the Unversity of Oxford, OX1 3TH, Oxford, United Kingdom.

${ }^{5}$ Nuffield Department of Clinical Neurosciences, John Radcliffe Hospital, University of Oxford, OX3 9DU, Oxford, United Kingdom.

*Corresponding author (auyqli@scut.edu.cn)

Copyright (c) 2014 IEEE. Personal use of this material is permitted. However, permission to use this material for any other purposes must be obtained from the IEEE by sending an email to pubs-permissions@ieee.org. keyboards and mice) to help people with neuromuscular impairments (such as patients with disturbance of consciousness, amyotrophic lateral sclerosis, and stroke) to better interact with their environment [2]. EEG-based HMIs, which are also called brain-computer interfaces (BCIs), are usually developed based on event-related potentials (ERPs), including slow cortical potentials (SCPs) [3], steady-state visually evoked potentials (SSVEPs) [4], P300 potentials [5], and sensorimotor rhythms (mu and beta rhythms) [6]. Hemodynamic signals, such as functional near-infrared spectroscopy (fNIRS), can also be used to establish BCIs, which can produce different commands by measuring the blood flow changes in the local capillary network caused by neuron firings [7], [8]. EOG signals associated with different eye movements, such as horizontal/vertical eye movement, gaze, and blink, are also widely used to develop HMIs [9]. EOG usually has a strong signal amplitude, which makes it relatively easy to detect and therefore suitable for developing HMIs for those with the ability to control their eye movements. To accommodate different requirements, many kinds of BCIs or EOG-based HMIs have been developed for real-world applications, such as text speller [10], device control [11] and game interactions [12]. These HMI systems can work in either a synchronous mode, which requires a cue/trigger to instruct the beginning of each mental task, or an asynchronous mode, which is more practical because it enables the user to start a mental task in a self-paced fashion [13].

Computers have become a pervasive part of modern life. Assisting users with limited motor functions to conveniently interact with computers is one meaningful and promising application of EEG- and EOG-based HMIs. The most commonly used applications of personal computer (PCs) include spellers, which enable the user to input text; web browsers, which enable the user to obtain information from the internet; email clients, which provide a medium for communication; and file explorers, which enable the user to manage and manipulate files stored in the computer. All these applications rely on an efficient button selection method, with which the user is able to provide sufficient different commands, and/or a two-dimensional (2-D) mouse movement control, which allows the user to move the mouse between two arbitrary positions. Furthermore, both methods should be able to run in an asynchronous mode just like a typical keyboard and mouse.

In recent years, contemporary researchers have developed various types of asynchronous BCI spellers [14]-[16] or BCI browsers [17]-[19] based on P300 potentials [14], [19], 
N200 potentials [15], SSVEPs [16], SCPs [17], $\mu$ rhythms [18], or combinations of P300 and SSVEPs [16]. EEG and fNIRS can also be combined to develop hybrid BCI systems [20]; for instance, M. J. Khan et al. developed an EEGfNIRS-based hybrid BCI with an accuracy of over $80 \%$ for four independent commands [21]. Another EEG-fNIRS-based hybrid BCI with 8 independent commands was developed by the same group for quadcopter control, in which four tasks, including mental arithmetic, mental counting, mental rotation, and word formation, were decoded using fNIRS, and four eye movements, including two eyeblinks, three eyeblinks, up/down eye movement, and left/right eye movement, were decoded using EEG [22]. In 2004, Wolpaw and McFarland reported the first non-invasive BCI-based 2-D mouse control method; the authors showed that two independent control signals could be derived by training the subjects to regulate mu and beta rhythms simultaneously[23]. In our previous study, an MIand P300-based 2-D BCI mouse was developed, with which a mouse can be moved from an arbitrary position to another arbitrary position [24]. However, because of the limited number of independent commands (e.g., fewer than 10 in [14], [21], [22]) or high nonstationary and low signal-to-noise ratio (SNR) characteristics of EEG, most of these asynchronous BCI systems were unable to achieve high information transfer rates (ITRs) in the control state and simultaneously keep low false-positive rates (FPRs) in the idle state [24], [25], or required intensive user training [23], which hindered the practical application of these asynchronous BCI systems.

Some researchers have also developed asynchronous HMI systems based on EOG [9], [26]-[28]. For example, Usakli and Gurkan applied four kinds of eye movement (up, down, left, and right) to control the movement of a cursor, and they applied double blink to select targets. In this manner, a virtual keyboard was developed [26]. Similarly, Lledó et al. developed an EOG-based web browser by using EOG signals associated with four different directions of eye movement (left, right, up, and down) and blink [27]. EOG signals associated with different eye movements have also been used to control an electric wheelchair [28] and a humanoid robot [9]. However, most of these EOG-based HMI systems still encountered the following two problems: (i) it is still difficult to quickly and accurately distinguish intended eye movements from unintended eye movements, and (ii) the number of available commands is usually equal to the types of eye movements, which is generally limited and insufficient to develop a complex HMI system, e.g., a speller. In our previous study, an asynchronous EOG speller was developed in which a flashing mechanism was integrated to provide time stamps for users to blink their eyes and to assist the detection of eye blinks [29]. However, few EEG- or EOG-based practical designs have been developed for the other three aforementioned applications, i.e., web browsers, e-mail clients, and file explorers, which require an effective asynchronous mouse control method. In addition, to the best of our knowledge, previous studies have not integrated these four applications (speller, web browser, e-mail client, and file explorer) together in a single system in a real PC, which is important and necessary for practical use.

In the current study, we present an EOG-based button selection method, an EEG- and EOG-based 2-D mouse movement control method, and an asynchronous hybrid BCI system that integrates four asynchronous subsystems-a speller, a web browser, an e-mail client, and a file explorer-based on these two methods. Specifically, four buttons corresponding to the four abovementioned subsystems are presented in the main graphical user interface (GUI) of the system, and all four of these buttons alternatively flash in a random order. To enter/use a specific subsystem, the user is required to select the corresponding button. In the current study, to select a button, the user blinks his/her eyes in synchrony with the stimulus onset, or flash, of the desired button. The GUI of the subsystem speller consists of forty-one randomly flashing buttons. The user can insert a character by selecting the corresponding button. In the subsystem of the browser/e-mail client/file explorer, the user can control the mouse horizontal movement by imagining hand movement and can control the mouse vertical movement, select/reject targets, or spell characters by selecting specific buttons on the GUI. In this manner, the user can open a desired web page, read the web content, or select an intended hyperlink or other target contained in the opened page using the web browser; receive or write e-mails using the e-mail client; and access his/her files and manage them using the file explorer. In this work, ten healthy subjects participated in an online experiment, and the experimental results showed that all of the subjects could use the proposed system to finish a comprehensive online task. We compared the experimental results to our previously developed P300- and MI-based hybrid BCI and some other asynchronous BCIs and found that superior performance was achieved, thus demonstrating the effectiveness of the proposed hybrid BCI.

The rest of this paper is organized as follows. Section II presents the methods, including data acquisition, the GUI, EOG-based button selection, EEG- and EOG-based mouse control, system calibration, and workload evaluation. Section III presents the experimental implementation and results, which is followed by Sections IV and V, where further discussion and conclusions are presented, respectively.

\section{Methods}

\section{A. Data Acquisition}

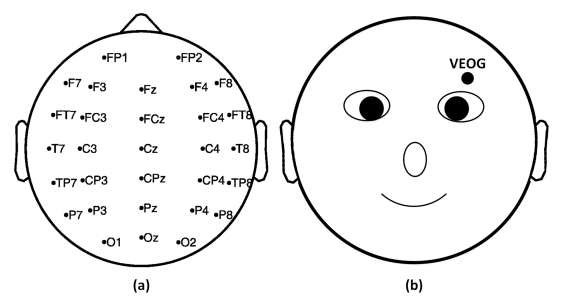

Fig. 1: Names and distribution of the electrodes for the EEG (a) and vertical EOG (b).

\section{B. GUI}

In this study, a NuAmps device (Neuroscan, Inc., Australia) and a 32-channel Quick-Cap (Neuroscan, Inc., Australia) are used to acquire the EEG/EOG signals. All electrodes are placed according to the international 10-20 system. Fig. 1(a) and (b) illustrate the placements of 30 electrodes and a monopolar electrode for recording EEG and vertical EOG 


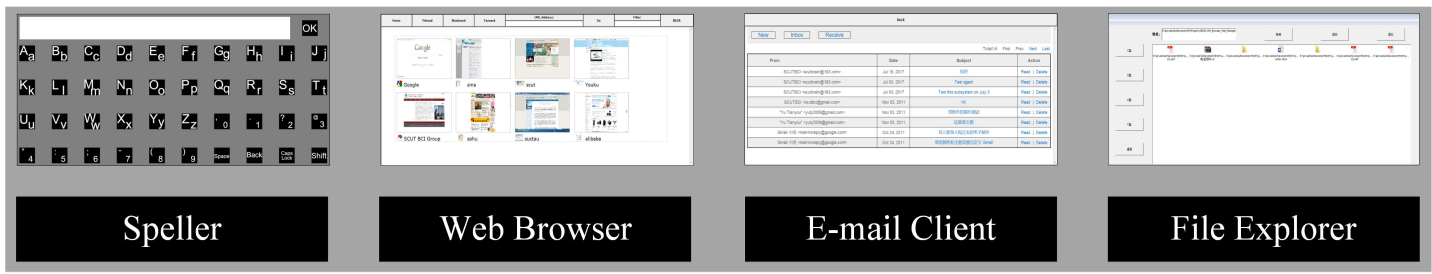

(a)

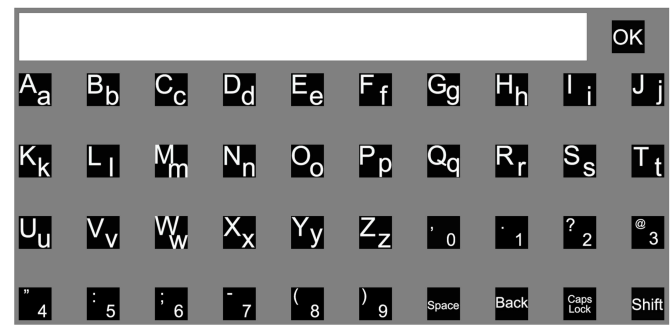

(b)

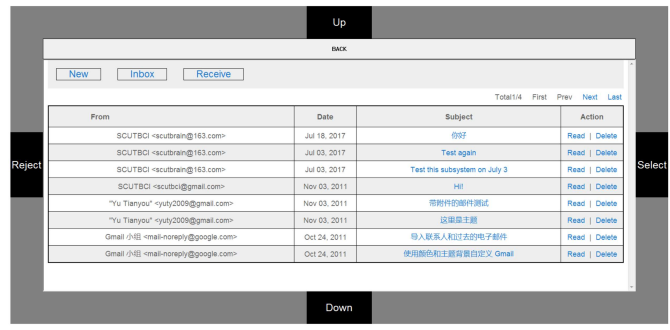

(d)

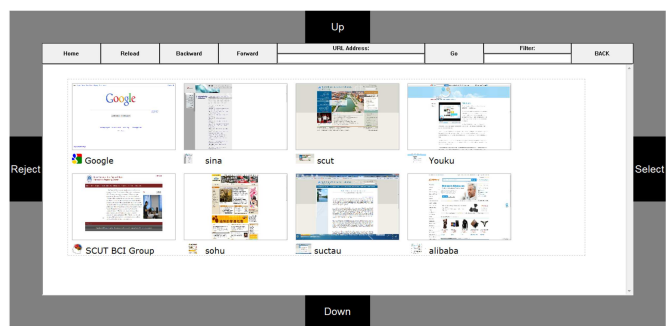

(c)

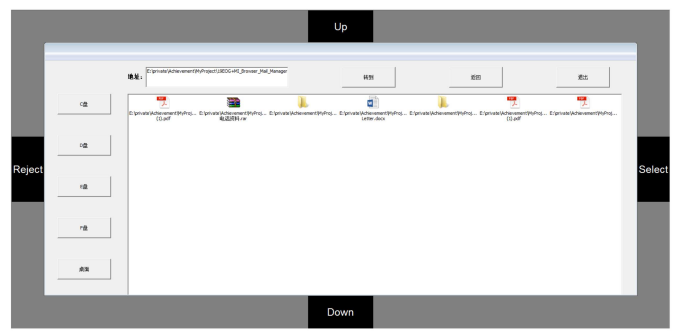

(e)

Fig. 2: GUIs for the (a) main interface of the hybrid BCI system, (b) speller, (c) web browser, (d) e-mail client, and (e) file explorer.

(VEOG), respectively. We only record single-channel VEOG because it has been suggested to be sufficient for blink detection [9], [29]. Although it is possible to detect intended eye movements from channels placed on the cortex, as suggested in [22], we use an additional monopolar channel placed near the upper eyelid to capture stronger blink-related VEOG. The ground electrode "GND" and reference electrode "A2" are separately attached to the forehead and right mastoid (not shown in the figure). During the experiments, the sampling rate is set to $250 \mathrm{~Hz}$, and for all electrodes used in this study, the impedance is maintained below $5 \mathrm{k} \Omega$.

As illustrated in Fig. 2, the hybrid BCI system consists of five GUIs corresponding to the main interface and four subsystems, i.e., the speller, web browser, e-mail client, and file explorer. Below, we describe each of these GUIs in detail.

\section{1) The main interface}

The GUI consists of four buttons corresponding to the abovementioned four subsystems (Fig. 2(a)). All four buttons are randomly and alternately intensified with a duration of $100 \mathrm{~ms}$ and a stimulus onset asynchrony (SOA) between two consecutive onsets of $250 \mathrm{~ms}$. SOA indicates the time interval between the onsets of any two adjacent button flashes/intensifications [30]. In this study, the procedure for each button flash includes changing the color of the button from black to green (flash onset), maintaining the green color for a duration of $100 \mathrm{~ms}$, and then changing the color of the button back to black (flash offset). The button flashes are performed round by round. In each round, each button flashes only once in a random order, and the difference between the onset times of two adjacent button flashes is $250 \mathrm{~ms}$ (SOA). The user can select a button to enter the corresponding subsystem. The button selection will be detailed later.

\section{2) The GUI for the speller}

As illustrated in Fig. 2(b), the GUI for the speller contains 41 buttons corresponding to 26 uppercase/lowercase letters, 10 Arabic numerals/punctuation marks, and five function buttons, including a "Space" button to input a space, a "Back" button to delete a character, a "CapsLock" button to switch between uppercase letters/punctuation marks and lowercase letters/Arabic numerals, a "Shift" button to switch between Chinese and English input methods, and an "OK" button to activate the main GUI with the spelled characters saved in a text file or to make the system go back to the previous subsystem with the spelled characters entered in the edit box. Specifically, if the speller GUI is activated by clicking an edit box, the "OK" button enters the spelled characters into the edit box and makes the system go back to the previous subsystem. If the speller GUI is activated by selecting the button "Speller" on the main interface, the "OK" button saves the spelled characters in a text file and saves the file in the root directory of the system. Once the speller subsystem is activated, all 41 buttons will flash in a random order with a duration and an SOA of $100 \mathrm{~ms}$ and $30 \mathrm{~ms}$, respectively. Note that an overlapping time of $70 \mathrm{~ms}$ exists between two adjacent button flashes, indicating that a maximum of 4 buttons will be colored green at the same time, although the onset times of these button flashes are different (30 ms apart from each other).

\section{3) The GUI for the web browser}

As illustrated in Fig. 2(c), the GUI for the web browser contains four flashing buttons around the client area, with a 
web page in the center and a navigation menu on the top. Specifically, these four buttons ("Up", "Down", "Select", and "Reject") flash in a random order with a duration and an SOA of $100 \mathrm{~ms}$ and $250 \mathrm{~ms}$, respectively. The user can select one of these buttons to make the mouse move up/down or to select/reject a target. In addition, the left/right movement of the mouse is controlled by left-/right-hand imagery. The imbedded web page contains eight predefined web sites. The developer can redefine these web sites according to the users suggestion. The navigation menu contains several practical function keys, including "Home", "Reload", "Backward", "Forward", URL address edit box, "Go", and a filter edit box. The last function key of the navigation menu, the "Back" key, takes the system back to the main interface. A demonstration of the use of the proposed web browser is presented in Fig. 3(a).

\section{4) The GUI for the e-mail client}

As shown in Fig. 2(d), the GUI and its control method for the e-mail client are similar to those for the web browser, which are described above. The "Back" function key, which is used to take the system back to the main interface, is placed on the top. The available e-mail communication operations include open an e-mail to read, create/reply to a new e-mail, forward an e-mail to others, and attach a file to the current e-mail. Fig. 3(b) shows a demonstration of the creation of a new e-mail using the proposed e-mail client.

\section{5) The GUI for the file explorer}

As shown in Fig. 2(e), the GUI and its control method for the file explorer are similar to the GUI and control method for the web browser and e-mail client. Specifically, all of the files and folders under the current directory are shown in the center of the file explorer client. Five buttons ("C" $\mathrm{C}$, "D", "E", "F", and "Desktop") located on the left side of the file explorer enable the file explorer to directly reach the corresponding directory. Four function keys, which consist of an edit box to activate the speller subsystem for new directory address typing, a "Go" button to access the specified directory, a "Previous" button to switch to the previous directory, and a "Back" button to make the system go back to the main interface, are placed on the top of the file explorer. Once the user clicks/selects a specified file listed in the file explorer client, the flashing buttons "Up", "Down", "Select", and "Reject" will be replaced by the "Open", "Paste", "Copy", and "Delete" buttons, respectively. The user can select one of these buttons to perform the corresponding manipulation. Once a manipulation is performed, the buttons will be translated back to the normal status. When a file (e.g., a pdf) is opened, the user can page up/down in the file or close it by selecting the flashing buttons "Up"//Down" and "Reject", respectively. Note that the mouse movement is invalid when a file is opened.

The system is started at the main interface (Fig. 2(a)). If one of the four buttons ("Speller", "Web Browser", "Email Client", and "File Explorer") is selected by the user, the corresponding subsystem will be activated. If the user selects an edit box provided by the hybrid system or contained in any web page, the system will switch to the speller subsystem (Fig. 2(b)) for the user to input text.

\section{EOG-based button selection}

In this study, button selection is achieved by using only blinkrelated EOG signals, as illustrated in Fig. 4. Specifically, the user can select a button by blinking synchronously with the flashes of this button; meanwhile, the vertical EOG signal from the monopolar electrode shown in Fig. 1(b) is recorded. Then, an online detection procedure is implemented to determine the target button for each round of button flashes.

\section{1) Feature extraction}

At the beginning of each detection, we first extract a segment of EOG signal from the monopolar EOG channel. Specifically, the data between 100 and $500 \mathrm{~ms}$ after each button flash onset are extracted. Then, we bandpass-filter the extracted segment of signal with a pass frequency band of 0.1-20 Hz. Next, a first-order difference operation is applied to achieve a feature vector. In this manner, we extract $N$ online feature vectors per round corresponding to $N$ button flashes.

\section{2) SVM classification}

A predefined support vector machine (SVM) classifier is applied to these online feature vectors to obtain $N$ SVM scores, among which we keep $n$ maximum scores and set the others to zero. Those $n$ buttons corresponding to the reserved $n$ maximum scores are regarded as precandidate target buttons. In the current study, we set $n$ to five for the speller GUI and to one for the other GUIs. This is because a single blink may be associated with several button flashes in the speller GUI because of its short SOA (30 ms), while in the other GUIs, a blink is usually only associated with one button flash, as their SOAs are long (250 ms).

\section{3) Waveform detection}

To detect whether the extracted online feature vectors contain a blink, we apply a waveform detection procedure to each of them simultaneously with the SVM classification. Specifically, a typical first-order differential EOG waveform $(X)$ consists of a peak and a valley. We first calculate a time duration $d$ and an energy $e$ for each feature vector $X$, as below:

$$
\left\{\begin{array}{l}
X=\left[x_{1}, x_{2}, \ldots, x_{99}\right] \\
d=t_{\text {valley }}-t_{\text {peak }}, e=\sum_{t=t_{\text {peak }}}^{t_{\text {valley }}}\left(x_{t}\right)^{2}
\end{array}\right.
$$

where $t_{\text {peak }}$ and $t_{\text {valley }}$ represent the time points of the valley and peak, respectively, and $d$ and $e$ indicate the speed and strength of the blink, respectively. Then, three predefined thresholds, including a minimum time duration threshold $T_{\min }$, a maximum time duration threshold $T_{\max }$, and a minimum energy threshold $E$, are used to complete the waveform detection. For a specific feature vector, if the time duration $t$ is between $T_{\min }$ and $T_{\max }$, and the energy $e$ is larger than $E$, then we obtain a result of 1 , which means the waveform detection for the corresponding button flash is passed; otherwise, a result of 0 is obtained, which means the waveform detection is unpassed for this button flash.

\section{4) Candidate target determination}

The buttons corresponding to nonzero SVM scores and nonzero waveform detection results are selected as candidate targets of the current round of button flashes.

\section{5) Decision making}

For the GUI of the file explorer, e-mail client, or web browser, the button that fulfills the conditions in step 4 is regarded as 


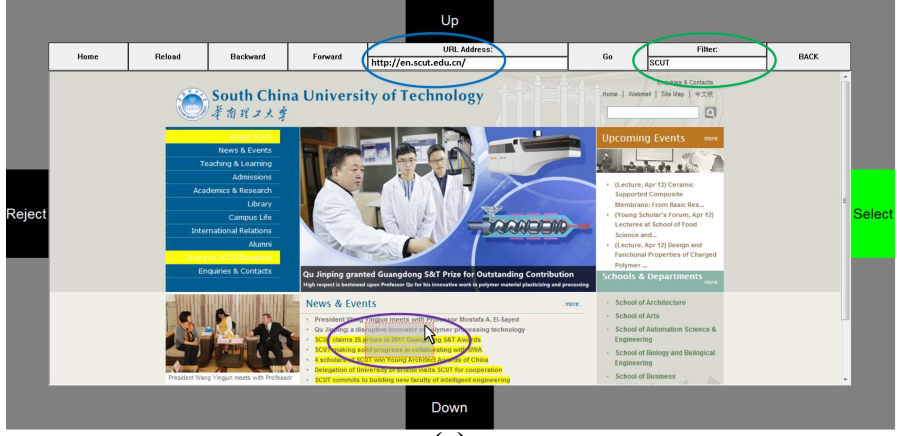

(a)

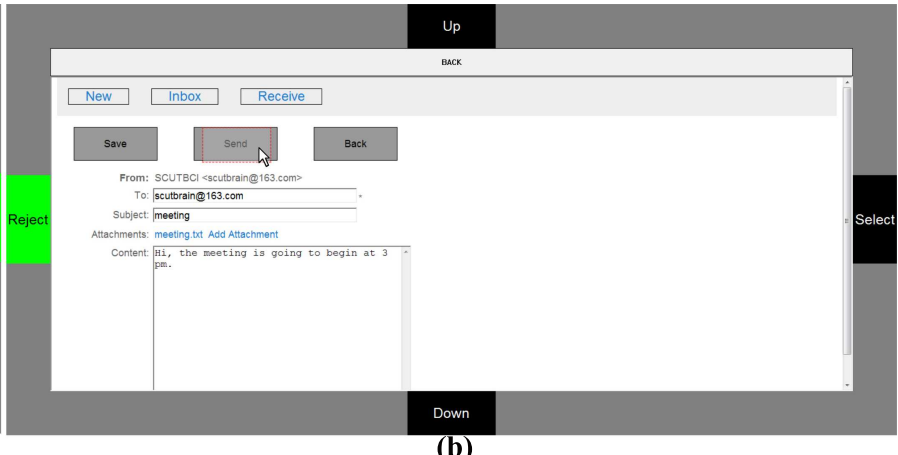

(b)

Fig. 3: (a) A demonstration of surfing the internet using the proposed web browser, in which the input URL address, input keyword for target filtering, the touched target, and the remaining targets after filtering are circled in blue, green, and purple and highlighted in yellow, respectively. (b) A demonstration of creating an email using the proposed email client.

the target button. For the GUI of the speller or main interface, the decision is made according to the results of the current and previous two rounds of button flashes. Specifically, if a button is identified as a candidate target at least twice in the latest three consecutive detections/rounds, then this button is selected as the target button of the current detection. If there are two or more buttons fulfilling this condition, then we calculate a value for each of these buttons by summarizing the SVM scores corresponding to the latest three detections/rounds and select the button with the maximum value as the target button. Otherwise, no target button is determined in the current detection. Setting different decision-making conditions for the GUIs of the speller subsystem and the other subsystems is due to their different SOAs. In the GUI of the speller subsystem containing 41 buttons, we set an extremely short SOA of $30 \mathrm{~ms}$ to achieve a high ITR in the control state, which at the same time makes it difficult to synchronize a blink with a specific button flash. As a result, we utilize the results of the current and previous two rounds of button flashes to determine a target button. However, the SOAs of the other GUIs containing only four buttons are $250 \mathrm{~ms}$, which are usually long enough to identify the target button according to a single blink in the current round of button flashes.

\section{EEG- and EOG-based mouse control}

In this study, mouse movement and target selection/rejection are achieved using the MI-related EEG and blink-related EOG signals, as shown in Fig. 4. Specifically, MI-related EEG is used to control the horizontal movement of the mouse, i.e., left/right, while blink-related EOG is used to control the vertical movement of the mouse, i.e., up/down, as well as to select/reject targets. The horizontal and vertical movements of the mouse, which are controlled by performing MI and blink tasks, are independent of each other.

\section{1) Mouse horizontal movement control}

The mouse position is updated every $200 \mathrm{~ms}$, in which the horizontal position of the mouse is updated according to the classification result of MI-related EEG signals. More specifically, a segment of $600 \mathrm{~ms}$ of EEG data is first extracted from every $200-\mathrm{ms}$ interval $(400 \mathrm{~ms}$ of data overlapped with the previous segment). Then, a common average reference (CAR) filter and an 8- to 13-Hz bandpass filter are successively applied to filter the extracted data. Next, a common spatial pattern (CSP) algorithm is utilized to extract the feature vector
[31]. After that, a pretrained SVM classifier is used to calculate an SVM score, i.e., $s_{k}$, for the extracted feature vector. Finally, the new horizontal position of the mouse is calculated as

$$
x_{k}=x_{k-1}+a\left[s_{k-2}+s_{k-1}+s_{k}\right]+b,
$$

where $a$ and $b$ are two parameters used to keep $\left(x_{k}-x_{k-1}\right)$ close to zero while the user is in an idle state [24]. Note that the user can perform an MI task to control the mouse horizontal movements at any time without attending to the button flashes because the detection of MI is independent of the flashes of the buttons.

\section{2) Mouse vertical movement control}

The vertical movement of the mouse is controlled by selecting the "Up"/"Down" button. Specifically, if the user selects the "Up"/"Down" button using the previously introduced button selection method, the mouse will move upward/downward with a fixed vertical (20 pixels per update). The mouse will not stop unless the "Reject" button is selected or a target is touched.

\section{3) Target selection or rejection}

Once a target is touched, the mouse will stop automatically. If the target is interesting, then the user can select the "Select" button to click and open it; otherwise, the user can select the "Reject" button to reject it. Once a target is rejected, the mouse will continue to move according to the previous movement state.

\section{E. Calibration}

Each subject is required to participate in a calibration procedure prior to his online experiment. In the calibration procedure, two SVM classifiers and three thresholds $\left(T_{\text {min }}\right.$, $T_{\max }$, and $E$ ) are determined for the online experiment.

First, each subject attends an MI training session including 60 trials, each of which consists of a $4 \mathrm{~s}$ left/right MI task. The collected EEG data are first filtered using a CAR filter and an 8- to $13-\mathrm{Hz}$ bandpass filter. Based on these filtered EEG data, a CSP transform matrix $W$ is constructed using the well-known joint diagonalization method [24]. Three components from both ends of $W$ are selected to extract the discriminative spatial patterns (feature vectors) for each MI class. In this manner, sixty 6-D feature vectors with label 1 (left-hand motor imagery) or -1 (right-hand motor imagery) are obtained from this training dataset. Using these feature 


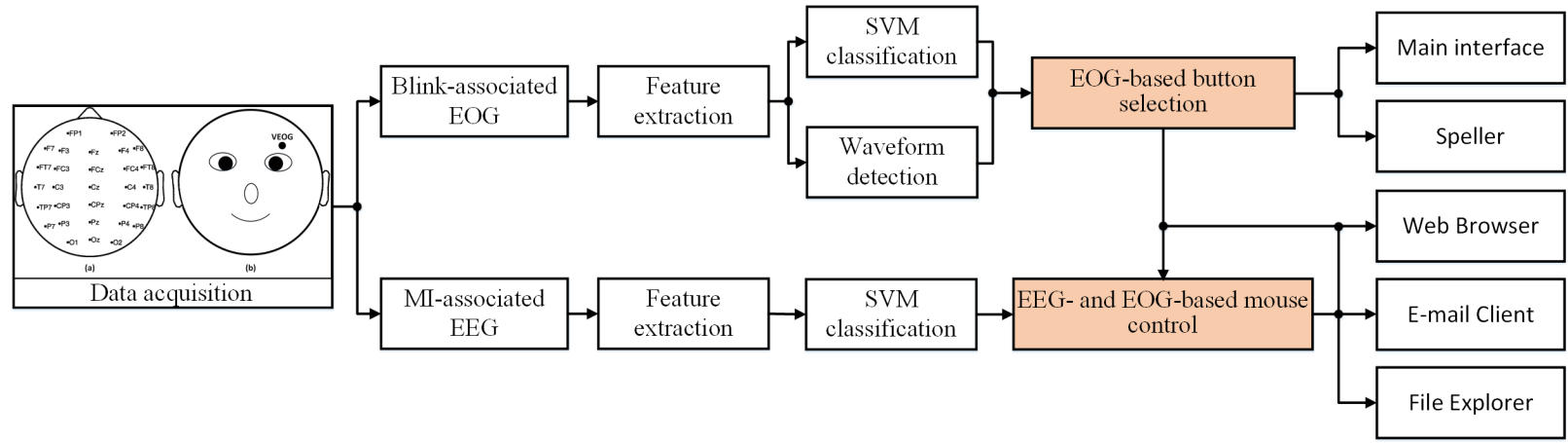

Fig. 4: Flowchart of the entire system. The EOG and EEG signals are acquired and fed into two different data processing procedures simultaneously. Specifically, based on the blink-related EOG signal, a button selection method is implemented and is used to operate the main interface as well as the speller. A mouse movement control method is implemented based on the blink-related EOG and MI-related EEG signals. With the collaboration of mouse movement control and button selection/rejection, the user can operate the web browser, e-mail client, and file explorer.

\begin{tabular}{|c|c|c|c|}
\hline & Experimer & paradigm & \\
\hline $\begin{array}{l}\text { 1. Surfing the internet using the } \\
\text { web browser subsystem. }\end{array}$ & $\begin{array}{l}\text { 2. Write a sentence in a text file } \\
\text { using the speller subsystem. }\end{array}$ & $\begin{array}{l}\text { 3. Manage the text file using the } \\
\text { explorer subsystem. }\end{array}$ & $\begin{array}{l}\text { 4. Send and receive an e-mail } \\
\text { with the file attached using the e- } \\
\text { mail client subsystem. }\end{array}$ \\
\hline $\begin{array}{l}\text { 1) Select the button "Web Browser" ; } \\
\text { 2) Move mouse and select a predefined } \\
\text { web; } \\
\text { 3) Determine a keyword, move mouse, } \\
\text { and select the filter; } \\
\text { 4) Input the keyword; } \\
\text { 5) Move mouse and select a target; } \\
\text { 6) Move mouse and select the button } \\
\text { "Back". }\end{array}$ & $\begin{array}{l}\text { 1) Select the button "Speller" ; } \\
\text { 2) Input a file name; } \\
\text { 3) Select the button "OK" to produce a } \\
\text { text file with the input file name; } \\
\text { 4) Input a sentence of "An EEG-and } \\
\text { EOG-based asynchronous hybrid BC } \\
\text { system." } \\
\text { 5) Keep in the idle state for about } 5 \mathrm{~min} \text {; } \\
\text { 6) Select the button "OK" to save the } \\
\text { sentence in the text file and back to the } \\
\text { initial interface. }\end{array}$ & $\begin{array}{l}\text { 1) Select the button "Explorer"; } \\
\text { 2) Find the file generated in the previous } \\
\text { subtask; } \\
\text { 3) Open it; } \\
\text { 4) Close it; } \\
\text { 5) Copy the file; } \\
\text { 6) Paste the file in the current directory; } \\
\text { 7) Back to the initial interface. }\end{array}$ & $\begin{array}{l}\text { 1) Select the button "E-mail Client" ; } \\
\text { 2) Select the button "New"; } \\
\text { 3) Enter the address of current account; } \\
\text { 4) Enter a subject; } \\
\text { 5) Add the file as an attachment; } \\
\text { 6) Write the e-mail; } \\
\text { 7) Send the e-mail; } \\
\text { 8) Back to the initial interface. } \\
\text { 9) Select the button "E-mail Client" ; } \\
\text { 10) Select the button "Receive" ; } \\
\text { 11) Open the e-mail send just now; } \\
\text { 12) Open the attached file; }\end{array}$ \\
\hline
\end{tabular}

(a)

(b)

(c)

(d)

Fig. 5: Paradigm of a trial in the online experiment. (a) Subtask I: Surf the internet (b) Subtask II: Write a sentence in a file. (c) Subtask III: Manage the file. (d) Subtask IV: Send and receive an e-mail.

vectors and the corresponding labels, a linear SVM classifier for the classification of MI-related EEG signals is trained.

Second, a training dataset of EOG is collected for each subject by using the speller GUI (Fig. 2(b)). The training session consists of 20 trials. Each trial consists of 10 rounds of button flashes. During this training session, the subject is required to blink his eyes synchronously with the flashes of the target buttons. Based on this dataset, a feature vector is extracted using the abovementioned feature extraction method for each button flash. In total, 200 feature vectors with label 1 are achieved for the target button flashes, and 8000 feature vectors with label -1 are achieved for the nontarget button flashes. Next, from among the 8000 nontarget feature vectors, we further select 200 feature vectors corresponding to the button flashes far away from the target button flashes as the negative training samples. Using these feature vectors and the corresponding labels, a linear SVM classifier for the classification of blink-related EOG signals is trained.

Third, we separately calculate 200 duration and energy values ( $d$ and $e$ in Eq. 1) using the above extracted 200 feature vectors labeled 1 . Then, we remove those values smaller than $\mu-3 \sigma$ or larger than $\mu+3 \sigma$, in which $\sigma$ and $\mu$ represent the standard deviation and expectation of these values, respectively. Next, we separately sort the rest of the energy and duration values in ascending order. Finally, we select the largest values of the bottom 5\% and 95\% among the sorted duration values as thresholds $T_{\min }$ and $T_{\max }$, respectively. Similarly, among the sorted energy values, we select the largest value of the bottom $5 \%$ as threshold $E$. These three thresholds are utilized to identify EOG waveforms corresponding to intended eye blinks and to simultaneously exclude waveforms corresponding to noise or some other types of eye movements such as looking up, down, left, and right. According to our experience, selection of such thresholds can achieve a satisfactory balance between intended blinks detection and noise or other types of eye movement exclusion.

\section{EXPERIMENTS AND RESULTS}

\section{A. Experimental process}

Ten healthy male subjects with normal or corrected-to-normal vision, aged from 23 to 28 years, took part in a comprehensive online experiment to evaluate the effectiveness of the proposed hybrid BCI. All subjects were required to sign informed consent forms before their experiments. Next, a 5min preparation session and a 20-min training session were performed sequentially. In the preparation session, the subject received instructions to familiarize him with the proposed hybrid system as well as the experimental procedure. During the training session, two SVM classifiers and three thresholds were obtained, as illustrated in Section II-E: Calibration. Then, 
TABLE I: Results obtained in Subtask I.

\begin{tabular}{|c|c|c|c|c|c|c|c|c|c|c|c|c|c|}
\hline Sub. & Trials & $\mathrm{N} 1$ & $\mathrm{ACC} 1(\%)$ & RT1 (s) & FPR (events/min) & $\mathrm{N} 2$ & $\mathrm{ACC} 2(\%)$ & RT2 (s) & $\mathrm{T} 2(\mathrm{~s})$ & N3 & $\operatorname{ACC} 3(\%)$ & $\mathrm{T} 3(\mathrm{~s})$ & $\mathrm{T}(\min )$ \\
\hline $\mathrm{S} 1$ & 5 & 6.4 & 96.88 & 1.59 & 0.23 & 5.4 & 96.30 & 5.99 & 32.32 & 6.4 & 100 & 18.48 & 2.68 \\
\hline S2 & 5 & 6.8 & 97.06 & 1.90 & 0 & 6.4 & 96.88 & 6.25 & 40 & 6.8 & 100 & 18.68 & 2.30 \\
\hline S3 & 5 & 6.2 & 100 & 1.68 & 0.2 & 6.8 & 94.12 & 5.75 & 39.1 & 6.2 & 100 & 23.88 & 3.29 \\
\hline S4 & 5 & 5.4 & 100 & 1.77 & 0.15 & 5 & 100 & 5.46 & 27.3 & 5.4 & 100 & 25.67 & 2.92 \\
\hline S5 & 5 & 7.4 & 97.30 & 1.34 & 0.26 & 6.8 & 94.12 & 6.11 & 41.55 & 7.4 & 100 & 18.26 & 3.11 \\
\hline S6 & 5 & 7.6 & 87.5 & 1.97 & 0.23 & 5.4 & 95.14 & 7.03 & 37.96 & 7.6 & 98.67 & 17.57 & 3.11 \\
\hline S7 & 5 & 5.4 & 95.67 & 2.00 & 0.16 & 6.2 & 92.86 & 6.80 & 42.16 & 6.4 & 100 & 19.44 & 2.96 \\
\hline S8 & 3 & 6.3 & 91.67 & 1.73 & 0.44 & 5.7 & 91.67 & 6.57 & 37.23 & 6.3 & 100 & 17.32 & 2.63 \\
\hline S9 & 4 & 8.5 & 93.61 & 2.35 & 0.28 & 5.25 & 93.92 & 5.92 & 31.08 & 8.5 & 97.5 & 20.76 & 3.79 \\
\hline $\mathrm{S} 10$ & 5 & 7.0 & 92.86 & 2.04 & 0.22 & 5.4 & 91.63 & 7.02 & 37.90 & $\begin{array}{l}0.5 \\
7.0\end{array}$ & 97.14 & 18.81 & 3.06 \\
\hline Avg & 4.7 & 6.7 & 95.26 & 1.84 & 0.22 & 5.84 & 94.66 & 6.29 & 36.66 & 6.8 & 99.33 & 19.89 & 2.99 \\
\hline
\end{tabular}

TABLE II: Results obtained in Subtask II.

\begin{tabular}{cccccccc}
\hline Sub. & Trials & N & $\begin{array}{c}\text { ACC } \\
(\%)\end{array}$ & $\begin{array}{c}\text { RT } \\
(\mathrm{s})\end{array}$ & $\begin{array}{c}\text { ITR } \\
(\mathrm{bit} / \mathrm{min})\end{array}$ & $\begin{array}{c}\text { FPR } \\
(\text { events/min })\end{array}$ & $\begin{array}{c}\mathrm{T} \\
(\mathrm{min})\end{array}$ \\
\hline S1 & 5 & 52 & 98.44 & 6.01 & 51.51 & 0 & 6.41 \\
S2 & 5 & 52 & 92.75 & 6.16 & 44.80 & 0 & 7.39 \\
S3 & 5 & 52 & 95.59 & 6.21 & 46.68 & 0.2 & 7.03 \\
S4 & 5 & 52 & 96.97 & 5.59 & 53.35 & 0 & 6.14 \\
S5 & 5 & 52 & 93.66 & 5.20 & 53.59 & 0 & 6.16 \\
S6 & 5 & 52 & 90.90 & 6.91 & 38.50 & 0.1 & 6.99 \\
S7 & 5 & 52 & 93.54 & 6.54 & 42.83 & 0.15 & 6.67 \\
S8 & 3 & 52 & 94.29 & 6.78 & 41.92 & 0 & 7.37 \\
S9 & 4 & 52 & 89.15 & 6.13 & 41.94 & 0 & 6.82 \\
S10 & 5 & 52 & 91.66 & 6.06 & 44.55 & 0 & 6.75 \\
\hline Avg & 4.7 & 52 & 93.70 & 6.16 & 45.97 & 0.05 & 6.77 \\
\hline
\end{tabular}

the online experimental session was conducted, in which all subjects were required to complete a comprehensive task five times (trials). However, due to personal reasons, S8 and S9 only finished 3 and 4 trials, respectively. As shown in Fig. 5, the task could be divided into four subtasks corresponding to four subsystems: (i) surfing the internet using the web browser subsystem, (ii) writing a sentence in a text file using the speller subsystem, (iii) managing the text file using the file explorer subsystem, and (iv) sending and receiving an e-mail with an attachment using the e-mail client subsystem. In each trial, the subjects performed all four subtasks without pausing, although they could rest at any time because the proposed integrated system had an asynchronous design. To complete the online experiment, each subject finished three to five trials with a break of 5 min between two trials. Below, the details of each subtask as well as the performance of the corresponding subsystem are presented.

1) Subtask I: Surfing the internet using the web browser

As illustrated in Fig. 5(a), during Subtask I, the subject needed to surf the internet using the web browser subsystem according to the following six sequential steps: 1) Activate the browser subsystem by selecting the "Web Browser" button. 2) Move the mouse and select a web site from the eight predefined web sites, which usually consists of many selectable links/targets. 3) Move the mouse and select the target filter to activate the speller subsystem. 4) Spell a keyword and return the system to the browser subsystem by selecting the "OK" button. Then, those targets containing the keyword remain, and other targets are filtered out. 5) Move the mouse and select an interesting target from the remaining targets (i.e., targets containing the keyword) to open the corresponding web page. 6) Return to the main interface.

2) Subtask II: Write a sentence in a file using the speller

During this subtask, all subjects were required to write a sentence in a text file and save it using the speller subsystem.
TABLE III: Results obtained in Subtask III.

\begin{tabular}{ccccccccc}
\hline Sub. & Trials & N1 & $\begin{array}{c}\text { ACC1 } \\
(\%)\end{array}$ & $\begin{array}{c}\text { RT } \\
(\mathrm{s})\end{array}$ & $\begin{array}{c}\text { N2 } \\
\text { ACC2 } \\
(\%)\end{array}$ & $\begin{array}{c}\text { T2 } \\
(\mathrm{s})\end{array}$ & $\begin{array}{c}\mathrm{T} \\
(\mathrm{min})\end{array}$ \\
\hline S1 & 5 & 7 & 96.15 & 1.53 & 7 & 100 & 20.91 & 2.62 \\
S2 & 5 & 7.2 & 100 & 1.49 & 7.2 & 100 & 21.53 & 2.76 \\
S3 & 5 & 8 & 93.33 & 1.60 & 8 & 100 & 24.21 & 3.44 \\
S4 & 5 & 7.8 & 97.44 & 1.50 & 7.8 & 100 & 19.64 & 2.75 \\
S5 & 5 & 7.4 & 94.60 & 1.84 & 7.4 & 100 & 18.34 & 2.49 \\
S6 & 5 & 7.8 & 95.28 & 2.13 & 7.8 & 100 & 17.55 & 2.56 \\
S7 & 5 & 9.4 & 91.23 & 1.81 & 9.4 & 100 & 18.66 & 3.21 \\
S8 & 3 & 7 & 93.33 & 1.79 & 7 & 96.67 & 20.89 & 2.64 \\
S9 & 4 & 7.5 & 93.29 & 1.95 & 7.5 & 96.57 & 22.03 & 3.00 \\
S10 & 5 & 7.8 & 95.27 & 1.96 & 7.8 & 94.81 & 23.46 & 3.30 \\
\hline Avg & 4.7 & 7.69 & 94.99 & 1.76 & 7.69 & 98.81 & 20.72 & 2.88 \\
\hline
\end{tabular}

More specifically, the subtask could be divided into the following six sequential steps (Fig. 5(b)): 1) Select the "Speller" button in the initial GUI. 2) Input a file name. 3) Select the "OK" button to produce a text file with the input file name. 4) Input the text "An EEG- and EOG-based asynchronous hybrid BCI system.". 5) Remain in the idle state for 5 min. 6) Select the "OK" button to save the sentence in the text file and return to the initial GUI.

\section{3) Subtask III: Manage the file using the file explorer}

In this subtask, all subjects were required to access a local path and manipulate (open, close, copy, and paste) the file generated in the previous subtask using the file explorer subsystem. More specifically, the subtask could be divided into the following seven sequential steps (Fig. 5(c)): 1) Select the "File Explorer" button in the initial GUI. 2) Access a specified directory to find the file generated in the previous subtask. 3) Open the file. 4) Close the file. 5) Copy the file. 6) Paste the file into the current directory. 7) Return to the initial GUI.

4) Subtask IV: Send and receive e-mail with attached file using the e-mail client

During this subtask, all subjects were requested to send a new e-mail with an attachment to the current account and then receive it and open the attachment using the e-mail client subsystem. As illustrated in Fig. 5(d), 12 sequential steps were contained in this subtask: 1) Select the "E-mail Client" button in the initial GUI. 2) Move the mouse to select the "New" button to create a new e-mail after the e-mail client is opened. 3) Enter the e-mail address of the current account ("scutbrain@163.com") into the address edit box. 4) Enter a subject, e.g., a simple word. 5) Select the "Add attachment" button and select the file generated in Subtask II as an attachment. 6) Input a simple sentence as the e-mail content. 7) Move the mouse to select the "Send" button to send the e-mail to the current account. 8) Return to the initial interface. 9) Select the "E-mail Client" button in the initial 
GUI again. 10) After the e-mail client is opened, move the mouse to select the "Receive" button to receive the e-mail sent in step 7. 11) Move the mouse and open the newly received e-mail. 12) Move the mouse and open the attached file.

\section{B. Experimental results}

While using the web browser in Subtask I, the user was required to reject targets of no interest by selecting the "Reject" button and to correct incorrectly selected targets by selecting the "Backward" button. To evaluate the performance of the web browser subsystem, we summarized several indicators, as shown in Table I, in which N1, N2, and N3 indicated the average numbers of button selections/rejections, characters of the keyword used to filter, and mouse movements, respectively; $\mathrm{ACC} 1, \mathrm{ACC} 2$, and $\mathrm{ACC} 3$ indicated the average accuracies of button selections/rejections, keyword spelling, and mouse movements, respectively; RT1 and RT2 represented the average response times of selecting/rejecting a target and spelling a character, respectively; and T2, T3, and T indicated the average times of text input, a mouse movement, and a complete subtask, respectively. FPR was the average falsepositive rate achieved while the user was in the idle state. Here, a mouse movement indicated moving the mouse from one item to another. If the subject did not finish a specific mouse movement within $60 \mathrm{~s}$, the system counted a failure of mouse movement. Otherwise, the system counted a successful mouse movement. The accuracy of mouse movements (ACC3) equaled the ratio of the successful mouse movement number to the total mouse movement number.

While using the speller in Subtask II, the subject was required to delete the incorrectly spelled characters using the "Back" button. To evaluate the performance of the speller subsystem, the average numbers of trials and spelled characters $(\mathrm{N})$, accuracy (ACC), response time of spelling a character (RT), ITR [14], FPR in step 5, and time to complete the subtask once (T, excluding the 5 min idle time in step 5) were calculated (Table II).

While using the file explorer in Subtask III, if an unintended operation was performed, e.g., an incorrectly directory was entered, the subject was required to remedy the mistake by selecting the "Previous" button. Table III shows the average results obtained in this subtask, including the average trials, numbers of selections/rejections (N1) and mouse movements (N2); accuracies of target selections/rejections (ACC1) and mouse movements (ACC2); and time spent performing a selection/rejection (RT), performing a mouse movement (T2), and completing the subtask $(\mathrm{T})$.

While using the e-mail client in Subtask IV, the user was required to reject targets of no interest by selecting the "Reject" button and to correct the incorrectly selected targets by selecting the "Backward" button. Table IV shows the results obtained in this subtask; the corresponding performance evaluation indices were the same as those used in Subtask I.

As shown in Tables I-IV, all ten subjects completed the surfing, spelling, file management, and e-mail sending and receiving subtasks, which were relatively comprehensive and consisted of multiple text inputs as well as mouse operations, with a satisfactory performance.

\section{Workload evaluation}

After the online experiment, the NASA-TLX questionnaire was completed by each subject independently. The questionnaire consists of 6 factors: mental demand, physical demand, temporal demand, performance, effort, and frustration level. Each factor has 20 intervals scored from 0 to 100, indicating different subjective workloads. A high score indicates a high subjective workload and vice versa [32]. Some studies have suggested that scores less than 50 [33] or 60 [34] can be regarded as acceptable. As shown in Fig. 6, the scores corresponding to the factors of mental demand and effort were higher than those corresponding to the other factors, suggesting that fatigue associated with the proposed control paradigm may be mainly caused by mental demand and the effort of blinking the eyes synchronously with the flashes of the target button. However, the average scores were less than 40 for all factors, suggesting that all ten subjects thought that the proposed EEG- and EOG-based hybrid system was acceptable.

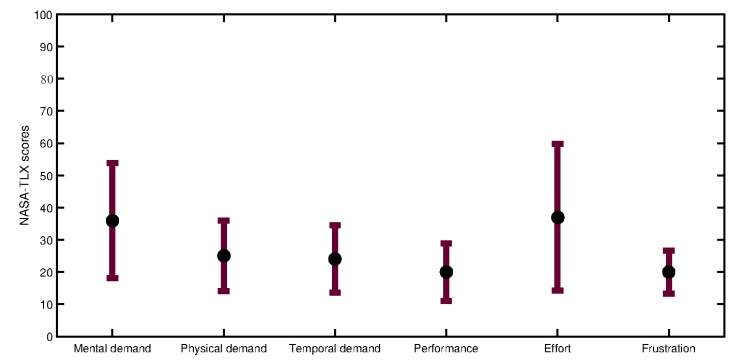

Fig. 6: The means and standard deviations of the NASA-TLX scores.

\section{Discussion}

Among the various applications available in PCs, spellers, web browsers, e-mail clients, and file explorers, which are best run in asynchronous mode, might be the four most commonly used applications. For these applications, a button selection method, which enables the users to select different buttons in an asynchronous mode, and a mouse movement control method, which enables the users to move the mouse between two arbitrary positions, are two essential tools. EEG- and EOG-based HCIs have promising potential to provide a new approach to help users with motor impairment to conveniently use these applications. However, asynchronous BCIs based on eventrelated potentials (e.g., P300 and SSVEPs) usually encounter a problem of low ITR or high FPR in the control or idle state because of the low SNRs of EEG [25]. In contrast, EOG-based HMIs usually have more reliable asynchronous performance because the signal patterns of EOG are more consistent and easier to detect. However, in studies of traditional EOG-based HMIs [9], [35], the number of commands is limited by the types of eye movements, which are usually insufficient to develop complicated systems with multiple commands, e.g., a speller. In addition, discriminating between intended and unintended eye movements in these EOG-based HMIs is difficult in some cases. To address these problems, we first proposed an EOG-based button selection method, in which we used button flashing to guide the users blinking, and the blinks were then easily detected and associated with the specific button. In this manner, the number of available commands was markedly in- 
TABLE IV: Results obtained in Subtask IV.

\begin{tabular}{|c|c|c|c|c|c|c|c|c|c|c|c|c|c|}
\hline Sub. & Trials & N1 & $\mathrm{ACC} 1(\%)$ & RT1 (s) & FPR (events/min) & $\mathrm{N} 2$ & $\operatorname{ACC} 2(\%)$ & RT2 (s) & $\mathrm{T} 2(\mathrm{~s})$ & N3 & $\operatorname{ACC} 3(\%)$ & $\mathrm{T} 3(\mathrm{~s})$ & $\mathrm{T}(\min )$ \\
\hline S1 & 5 & 25.2 & 97.62 & 1.45 & 0.09 & 32.4 & 96.91 & 6.01 & 194.72 & 25.2 & 100 & 22.53 & 13.32 \\
\hline $\mathrm{S} 2$ & 5 & 17.8 & 95.51 & 1.31 & 0.23 & 23.2 & 95.69 & 6.69 & 155.17 & 17.8 & 100 & 27.09 & 11.01 \\
\hline $\mathrm{S} 3$ & 5 & 18.8 & 96.81 & 1.47 & 0.12 & 25 & 96 & 6.07 & 151.63 & 18.8 & 100 & 25.11 & 10.86 \\
\hline S4 & 5 & 19.4 & 95.88 & 1.66 & 0.11 & 25.6 & 95.31 & 7.00 & 179.26 & 19.4 & 100 & 29.06 & 12.92 \\
\hline S5 & 5 & 19.6 & 94.90 & 1.39 & 0.07 & 23.8 & 94.96 & 6.43 & 152.94 & 19.6 & 100 & 22.50 & 10.35 \\
\hline S6 & 5 & 22.4 & 92.11 & 1.84 & 0.53 & 20.8 & 96.12 & 6.40 & 133.20 & 22.4 & 100 & 21.20 & 10.82 \\
\hline S7 & 5 & 20.4 & 88.57 & 1.92 & 0.37 & 20.6 & 92.51 & 6.45 & 132.87 & 20.4 & 100 & 25.96 & 11.69 \\
\hline S8 & 3 & 17.3 & 94.76 & 1.83 & 0.34 & 21 & 89.09 & 6.74 & 141.57 & 17.3 & 97.92 & 21.66 & 9.14 \\
\hline S10 & 5 & 20.8 & 92.68 & 1.41 & 0.32 & 23.8 & 92.75 & 5.97 & 142.00 & 20.8 & 96.47 & 21.16 & 10.19 \\
\hline Avg & 4.7 & 20.7 & 94.15 & 1.61 & 0.26 & 23.67 & 94.07 & 6.42 & 151.47 & 20.7 & 99.24 & 23.58 & 11.13 \\
\hline
\end{tabular}

creased, and simultaneously, based on the synchrony between the blink and the button flashing, the intended and unintended blinks could be more easily distinguished. Next, we proposed an EEG- and EOG-based asynchronous mouse control method, in which the mouse horizontal movement was controlled by MI-related EEG signals and the mouse vertical movement and target selection/rejection were controlled by blink-related EOG signals. Compared with pure MI-based 2-D mouse control method in [23], the training is much easier in the current study. Compared with pure P300/SSVEP/blink-based 2-D mouse control method in which the mouse can only be controlled discretely with many unreachable positions (will be detailed later), the proposed method allows mouse movement between arbitrary positions. Compared with the method proposed in our previous study [24], superior performance was achieved in the current study and this improvement mainly occurred because blink-related EOG presents a more significant signal pattern than P300 potential and is therefore much easier to detect online in asynchronous mode. Based on the proposed button selection and 2-D mouse control methods, we further developed an asynchronous hybrid system integrating a speller, a web browser, an e-mail client, and a file explorer, which enabled the user to input text, surf the internet, send/receive e-mails, and manage files in his/her PC without any body movements.

An effective asynchronous mouse control method is necessary for a practical HMI-based web browser, e-mail client, or file explorer. Particularly, arbitrary point-to-point control is an extremely important characteristic of mouse control, which requires two independent or at least nearly independent signals to separately control the horizontal and vertical movements of the mouse. In the current study, we used MI-related EEG and blink-related EOG signals to control the horizontal and vertical movements of the mouse, which produced much better performance than in our previous study [24]. This improvement mainly occurred because blink-related EOG presents a more significant signal pattern than P300 potential and is therefore much easier to detect online in asynchronous mode. The horizontal and vertical movements of the mouse can also be controlled by using EOG only, for example, by adding two additional buttons, shown in Fig. 2(c)-(e), to control the left and right movements of the mouse. However, several problems might be encountered with this method. First, it will lead to unsmooth and zigzagging mouse movement with many unreachable positions. As shown in Fig. 7, with this pure EOG-based control method, the mouse can only be moved toward one of eight possible positions (e.g., gray points) from the current position (red point), and any other positions (e.g., blue points) that are not located in the zigzag-like route (i.e., in directions 1-8) will be unreachable. In addition, the number of unreachable positions increases with the increased predefined movement speed and response time of button selection/rejection. Here the horizontal and vertical distances between two positions, i.e., $d_{x}$ and $d_{y}$, are determined based on the predefined horizontal and vertical speeds (e.g., 20 pixels/update) and the response time of selecting a button (e.g., $1.84 \mathrm{~s})$. In contrast, we utilized MI to control the horizontal movement of the mouse with a relatively short update time (200 ms) and a flexible step size in each update depending on the calculated SVM score (see Eq. 2). In this matter, the mouse movement status can be changed every $200 \mathrm{~ms}$ (compared with $1.84 \mathrm{~s}$ ) and the horizontal movement speed can be very large if the position of the target is far away from the current position of the mouse and vice versa, resulting in many more touchable positions and a smoother and more efficient 2-D mouse control. Second, the horizontal and vertical movement of the mouse cannot be controlled simultaneously by using EOG only. However, it is possible for the user to achieve this simultaneous movement by using both MI and EOG as they are essentially two different signals. Last, adding two additional buttons will either increase the RT of selecting/rejecting a target and the FPR during the idle state or decrease the accuracy of target selection/rejection. Specifically, if the SOA is kept at $250 \mathrm{~ms}$ after adding two more buttons, the time duration of a round of button flashes will increase from $1 \mathrm{~s}$ to $1.5 \mathrm{~s}$, which will result in an increased RT of button selection. However, the accuracy of button selection will decrease if the SOA is reduced because a single blink could be associated with a nontarget button flash more easily with a short SOA.

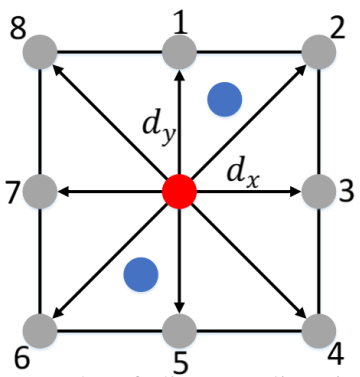

Fig. 7: Illustration example of discrete direction control of mouse, i.e., by EOG-based HMI.

To evaluate the performance of the proposed asynchronous hybrid system, a comprehensive online experiment was con- 
ducted with ten healthy subjects, in which each subject was required to complete four subtasks: surfing the internet (Subtask I), writing a sentence in a text file (Subtask II), managing the text file (Subtask III) and sending and receiving an e-mail with an attachment (Subtask IV). The experimental results shown in Tables I-IV were compared with those achieved in our previous studies [31], [40], [41], in which very similar experiments were conducted, as well as some other existing asynchronous BCI studies [9], [14]-[16], [36]-[39], [42], in which similar measurements were provided, to evaluate the effectiveness of the proposed hybrid system.

While using the web browser to surf the internet in Subtask I, we achieved a superior performance compared with our previous study [31], in which the mouse movement and target selection/rejection were achieved based on the combination of MI and P300. Specifically, the average time to complete a trial consisting of 6 steps was 3 min, which was 67.08 s shorter than that reported in [31], in which the average time to complete a trial consisting of only 4 steps was 247.08 s. Furthermore, the average RT for spelling a character was $6.29 \mathrm{~s}$, which was much shorter than the $13.6 \mathrm{~s}$ achieved in [31]. The average accuracy of target selection/rejection was also improved from $93.21 \%$ in [31] to $95.26 \%$ in the current study. During the mouse vertical movement control, we achieved a short RT (approximately 1-2 s) for selecting the "Up"/“Down" button and a relatively low average FPR of 0.22 event/min when the user was in the control and idle states. However, the RT to select the "Up"/"Down" button in [31] was as long as 12 $\mathrm{s}$, which was mainly because a restricted condition was set in detecting P300 potentials asynchronously to maintain an acceptable FPR.

While using the speller to spell characters in Subtask II, the achieved average accuracy, practical ITR, and FPR were $93.70 \%, 45.97 \mathrm{bits} / \mathrm{min}$, and 0.05/min, respectively. Compared with the benchmark results of previous asynchronous EOG[9], [36], [37] and EEG-based spellers [14]-[16], [38], [39], a superior performance was obtained in this study in terms of the number of available commands, accuracy, ITR, FPR, and other measures, as also demonstrated in our previous study [29].

While using the file explorer to manage the text file in Subtask III, we achieved an average accuracy of $94.99 \%$ with an RT of $1.76 \mathrm{~s}$ to perform a target selection/rejection and an average time of $20.72 \mathrm{~s}$ with an accuracy of $98.81 \%$ to complete a mouse movement between two targets. Superior performance was achieved compared with our previous work [40], in which a hybrid BCI based on P300 and MI was applied to design a file explorer and achieved an average time of nearly $1 \mathrm{~min}$ to complete a target selection/rejection and mouse movement.

While using the e-mail client to send and receive an e-mail with a file attached in Subtask IV, the achieved performance was comparable to that achieved in Subtask I. Specifically, the average accuracy and RT to make a target selection/rejection were $94.15 \%$ and $1.61 \mathrm{~s}$, the average accuracy and RT to spell a character were $94.07 \%$ and $6.42 \mathrm{~s}$, and the average time and accuracy to complete a mouse movement were 23.58 $\mathrm{s}$ and $99.24 \%$, respectively. Compared with the hybrid BCI- based e-mail client utilizing MI and P300 developed in our previous study [41], superior performance was achieved in this study. In [41], the average times to spell a character and complete a mouse movement were $12.44 \mathrm{~s}$ and $39.85 \mathrm{~s}$, respectively. Gannouni et al. developed a brain-controlled email client based on P300 named b-mail, in which different P300 commands were mapped to different actions of the email client [42]. With b-mail, the user might type up to 8 characters per minute, representing relatively low efficiency.

The integration of the four different but closely related systems of a speller, web browser, e-mail client, and file explorer is another innovation of this paper. For ease of use, it is necessary to integrate these systems as in a real PC. However, to the best of our knowledge, this is the first BCIbased study to integrate several commonly used interaction systems into a single system, which provides asynchronous functions of text input, internet surfing, e-mail communication, and file management.

Theoretically, the proposed EEG- and EOG-based hybrid BCI can also be used by patients suffering from a motor disability who can still control eye activities, such as those with Parkinsons, quadriplegia, motor neuron disease (MND), or spinal cord injury (SCI), although the performance with these patients requires further investigation. In addition, the health states of such patients may fluctuate, thus necessitating more adaptive detection methods/thresholds. However, for severely paralyzed patients with difficulty controlling their eye activities, such as patients suffering from central oculomotor disturbances and/or nystagmus due to neurodegeneration or brain lesions, the applicability of the proposed approach might be limited.

Another important issue for patients when using the proposed hybrid system is fatigue. The design of the proposed control paradigm requires users to pay attention to the flashes of the target button and blink their eyes synchronously with these flashes, which may be mentally demanding for the users, as suggested in Fig. 6. Compared with healthy subjects, patients may experience more difficulty when performing this task. To address this issue in the future, the potential solutions include: i) increasing the training time to allow the patients to become more familiar with the proposed system as well as its operation method; ii) increasing the time of SOA and/or reducing the number of buttons in the speller GUI to simplify the operation method; and iii) optimizing the detection method to render it more adaptive to the fluctuating health states of the patients.

\section{CONCLUSION}

This report describes an asynchronous hybrid BCI system using MI-related EEG and blink-related EOG signals. The proposed system consists of a web browser, speller, file explorer, and e-mail client, and all of these subsystems can work in asynchronous mode. While using the system, the user can control the mouse horizontal movement using MI-related EEG and can control the mouse vertical movement, select/reject targets, or spell characters using blink-related EOG. Based on the proposed system, the user can surf the internet, spell characters, access and manipulate files, and communicate with 
others by e-mail. In our online experiment, all ten subjects completed the comprehensive task with a satisfactory asynchronous performance in terms of high accuracy, short RT, and relatively low FPR. Therefore, the proposed system is effective. In the future, we plan to apply the proposed hybrid system for use by severely paralyzed patients.

\section{ACKNOWLEDGEMENTS}

Y. Li and S. He proposed the study paradigm and designed the system and experiments; S. He, Y. Zhou, T. Yu, R. Zhang, and Q. Huang implemented the system, conducted the experiments and analyzed the data; S. He, Y. Li, Z. Gu, and Z. L. Yu jointly wrote the manuscript; and H. Tan, L. Chuai and M. U. Mustafa revised and smoothed the manuscript.

\section{REFERENCES}

[1] B. A. Myers, "A brief history of human-computer interaction technology," Interactions, vol. 5, no. 2, pp. 44-54, Mar. 1998.

[2] J. R. Wolpaw, N. Birbaumer, D. J. McFarland, G. Pfurtscheller, and T. M. Vaughan, "Brain-computer interfaces for communication and control," Clin. Neurophysiol., vol. 113, no. 6, pp. 767-791, Jun. 2002.

[3] E. Donchin and D. B. D. Smith, "The contingent negative variation and the late positive wave of the average evoked potential," Electroenceph. Clin. Neurophysiol., vol. 29, no. 2, pp. 201-203, Aug. 1970.

[4] M. Middendorf, G. McMillan, G. Calhoun, and K. S. Jones, "Braincomputer interfaces based on the steady-state visual-evoked response," IEEE Trans. Rehabilitation Eng., vol. 8, no. 2, pp. 211-214, Jun. 2000

[5] A. Turnip and K.-S. Hong, "Classifying mental activities from EEGP300 signals using adaptive neural network," International Journal of Innovative Computing, Information and Control, vol. 8, no. 9, pp. 64296443, 2012.

[6] G. Pfurtscheller, C. Brunner, A. Schlögl, and F. H. Lopes da Silva, "Mu rhythm (de) synchronization and EEG single-trial classification of different motor imagery tasks," Neuroimage, vol. 31, no. 1, pp. 153-159, May 2006.

[7] K.-S. Hong and M. J. Khan, "Hybrid brain-computer interface techniques for improved classification accuracy and increased number of commands: A review," Frontiers in Neurorobotics, vol. 11, no. 35, 2017.

[8] M. J. Khan, U. Ghafoor, and K.-S. Hong, "Early detection of hemodynamic responses using EEG: A hybrid EEG-fNIRS study," Frontiers in Human Neuroscience, vol. 12, no. 479, Nov. 2018.

[9] J. Ma, Y. Zhang, A. Cichocki, and F. Matsuno, "A novel EOG/EEG hybrid human-machine interface adopting eye movements and ERPs: Application to robot control," IEEE Trans. Biomed. Eng., vol. 62, no. 3, pp. 876-889, Mar. 2015.

[10] N. Birbaumer, N. Ghanayim, T. Hinterberger, I. Iversen, B. Kotchoubey, A. Kübler, J. Perelmouter, E. Taub, and H. Flor, "A spelling device for the paralysed," Nature, vol. 398, no. 6725, pp. 297-298, Mar. 1999.

[11] B. Rebsamen, C. Guan, H. Zhang, C. Wang, C. Teo, M. H. Ang, and E. Burdet, "A brain controlled wheelchair to navigate in familiar environments," IEEE Trans. Neural Syst. Rehab. Eng., vol. 18, no. 6, pp. 590-598, Jun. 2010.

[12] E. C. Lalor, S. P. Kelly, C. Finucane, R. Burke, R. Smith, R. B. Reilly, and G. Mcdarby, "Steady-state VEP-based brain-computer interface control in an immersive 3D gaming environment," EURASIP Journal on Applied Signal Processing, vol. 2005, pp. 3156-3164, Jan. 2005.

[13] G. Townsend, B. Graimann, and G. Pfurtscheller, "Continuous EEG classification during motor imagery-simulation of an asynchronous BCI," IEEE Trans. Neural Syst. Rehabil. Eng., vol. 12, no. 2, pp. 258-265, Jun. 2004.

[14] H. Zhang, C. Guan, and C. Wang, "Asynchronous P300-based braincomputer interfaces: A computational approach with statistical models," IEEE Trans. Biomed. Eng., vol. 55, no. 6, pp. 1754-1763, Jun. 2008.

[15] D. Zhang, H. Song, H. Xu, W. Wu, S. Gao, and B. Hong, "An N200 speller integrating the spatial profile for the detection of the non-control state," J. Neural Eng., vol. 9, no. 2, p. 026016, Mar. 2012.

[16] R. C. Panicker, S. Puthusserypady, and Y. Sun, "An asynchronous P300 BCI with SSVEP-based control state detection," IEEE Trans. Biomed. Eng., vol. 58, no. 6, pp. 1781-1788, Jun. 2011.

[17] M. M. Moore, "Real-world applications for brain-computer interface technology," IEEE Trans. Neural Syst. Rehab. Eng., vol. 11, no. 2, pp. 162-165, Jun. 2003.
[18] A. A. Karim, T. Hinterberger, and J. Richter, "Neural internet: Web surfing with brain potentials for the complete-ly paralyzed," Neurorehabil. Neural Repair, vol. 20, no. 4, pp. 508-515, Dec. 2006.

[19] E. M. Mugler, C. A. Ruf, S. Halder, M. Bensch, and A. Kübler, "Design and implementation of a P300-based brain-computer interface for controlling an internet browser," IEEE Trans. Neural Syst. Rehabil. Eng., vol. 18, no. 6, pp. 599-609, Dec. 2010.

[20] K.-S. Hong, M. J. Khan, and M. J. Hong, "Feature extraction and classification methods for hybrid fNIRS-EEG brain-computer interfaces," Frontiers in Human Neuroscience, vol. 12, no. 246, pp. 1-25, June 2018.

[21] M. J. Khan, M. J. Hong, and K.-S. Hong, "Decoding of four movement directions using hybrid NIRS-EEG brain-computer interface," Frontiers in Human Neuroscience, vol. 8, no. 244, 2014.

[22] M. J. Khan and K.-S. Hong, "Hybrid EEG-fNIRS-based eight command decoding for BCI: Application to quadcopter control," Frontiers in Neurorobotics, vol. 11, no. 6, 2017.

[23] J. R. Wolpaw and D. J. McFarland, "Control of a two-dimensional movement signal by a noninvasive brain-computer interface in humans," Proceedings of the national academy of sciences, vol. 101, no. 51, pp. 17849-54, Dec. 2004.

[24] Y. Li, J. Long, T. Yu, Z. Yu, C. Wang, H. Zhang, and C. Guan, "An EEGbased BCI system for asynchronous 2-D cursor control by combining mu/beta rhythm and P300 potential," IEEE Trans. Biomed. Eng., vol. 57, no. 10, pp. 2945-2504, Oct. 2010.

[25] F. Lotte, M. Congedo, A. Lécuyer, F. Lamarche, and B. Arnaldi, "A review of classification algorithms for EEG-based brain computer interfaces," J. Neural Eng., vol. 4, no. 2, pp. R1-R13, Jan. 2007.

[26] A. B. Usakli and S. Gurkan, "Design of a novel efficient humancomputer interface: An electrooculagram based virtual keyboard," IEEE Trans. Instrum. Meas., vol. 59, no. 8, pp. 2099-2108, Aug. 2010.

[27] L. D. Lledó, A. Úbeda, E. Iáñez, and J. M. Azorín, "Internet browsing application based on electrooculography for disabled people," Expert Systems with Applications, vol. 40, pp. 2640-2648, 2013.

[28] R. Barea, L. Boquete, M. Mazo, and E. Lopez, "System for assisted mobility using eye movements based on electrooculography," IEEE Trans. Neural Syst. Rehabil. Eng., vol. 10, no. 4, pp. 209-218, Dec. 2002.

[29] S. He and Y. Li, "A single-channel EOG-based speller," IEEE Trans. Neural Syst. Rehabil. Eng., vol. PP, no. 99, Jul. 2017.

[30] J. E. Anderson and P. J. Holcomb, "Auditory and visual semantic priming using different stimulus onset asynchronies: An event-related brain potential study," Psychophysiology, vol. 32, no. 2, pp. 177-90, Mar. 1995.

[31] T. Yu, Y. Li, J. Long, and Z. Gu, "Surfing the internet with a BCI mouse," J. Neural Eng., vol. 9, no. 3, pp. 1-14, May 2012.

[32] S. G. Hart, L. E. Staveland, "Development of NASA-TLX (Task Load Index): Results of empirical and theoretical research," Adv. Psychol., vol. 52 , pp. $139-183,1988$

[33] M. H. R. Eitrheim and A. Fernandes, "The NASA Task Load Index for rating workload acceptability," HFES Europe 2016.

[34] P. T. McCabe, "Contemporary Ergonomics 2003," CRC Press, 2002.

[35] J. F. Wu, A. M. S. Ang, K. M. Tsui, H. C. Wu, Y. S. Hung, Y. Hu, J. N. F. Mak, S. C. Chan, and Z. G. Zhang, "Efficient implementation and design of a new single-channel electrooculography-based humanmachine interface system," IEEE Trans. Circuits Systems II: Express Briefs, vol. 62, no. 2, pp. 179-183, Feb. 2015.

[36] S. L. Wu, L. D. Liao, S. W. Lu, W. L. Jiang, S. A. Chen, and C. T. Lin, "Controlling a human-computer interface system with a novel classification method that uses electrooculography signals," IEEE Trans. Biomed. Eng., vol. 60, no. 8, pp. 2133-2141, Aug. 2013.

[37] C. C. Postelnicu and D. Talaba, "P300-based brain-neuronal computer interaction for spelling applications," IEEE Trans. Biomed. Eng., vol. 60, no. 2, pp. 534-543, Feb. 2013.

[38] F. Aloise, F. Schettini, P. Aricò, F. Leotta, S. Salinari, D. Mattia, F. Babiloni, and F. Cincotti, "P300-based brain-computer interface for environmental control: An asynchronous approach," J. Neural Eng., vol. 8, no. 2, pp. 025025, Mar. 2011.

[39] M. Schreuder, T. Rost, and M. Tangermann, "Listen, you are writing! Speeding up online spelling with a dynamic auditory BCI," Front. Neurosci., vol. 5, p. 112, Oct. 2011.

[40] L. Bai, T. Yu, and Y. Li, "A brain computer interface-based explorer," J. Neurosci. Methods, vol. 244, pp. 2-7, 2015.

[41] T. Yu, Y. Li, J. Long, and F. Li, "A hybrid brain-computer interfacebased mail client," Comput. Math. Methods Med., vol. 2013, Apr. 2013.

[42] S. Gannouni, N. Alrayes, A. A. A. Alsaudi, and A. Alabdulkareem, "B-mail: A brain-controlled mail client," Journal of Digital Information Management, vol. 9, no. 4, pp. 167-170, Aug. 2011. 